\title{
Applying Softcomputing for Copper Recovery in Leaching Process
}

\author{
Claudio Leiva, ${ }^{1}$ Víctor Flores, ${ }^{2}$ Felipe Salgado, ${ }^{1}$ Diego Poblete, ${ }^{1}$ and Claudio Acuña ${ }^{3}$ \\ ${ }^{1}$ Department of Chemical Engineering, Universidad Católica del Norte, Angamos Av. 0610, Antofagasta, Chile \\ ${ }^{2}$ Department of Computing \& Systems Engineering, Universidad Católica del Norte, Angamos Av. 0610, Antofagasta, Chile \\ ${ }^{3}$ Department of Chemical and Environmental Engineering, Universidad Técnica Federico Santa María, Valparaíso, Chile
}

Correspondence should be addressed to Víctor Flores; vflores@ucn.cl

Received 5 May 2017; Revised 10 October 2017; Accepted 30 October 2017; Published 5 December 2017

Academic Editor: Jezreel Mejia-Miranda

Copyright (C) 2017 Claudio Leiva et al. This is an open access article distributed under the Creative Commons Attribution License, which permits unrestricted use, distribution, and reproduction in any medium, provided the original work is properly cited.

\begin{abstract}
The mining industry of the last few decades recognizes that it is more profitable to simulate model using historical data and available mining process knowledge rather than draw conclusions regarding future mine exploitation based on certain conditions. The variability of the composition of copper leach piles makes it unlikely to obtain high precision simulations using traditional statistical methods; however the same data collection favors the use of softcomputing techniques to enhance the accuracy of copper recovery via leaching by way of prediction models. In this paper, a predictive modeling contrasting is made; a linear model, a quadratic model, a cubic model, and a model based on the use of an artificial neural network (ANN) are presented. The model entries were obtained from operation data and data of piloting in columns. The ANN was constructed with 9 input variables, 6 hidden layers, and a neuron in the output layer corresponding to copper leaching prediction. The validation of the models was performed with real information and these results were used by a mining company in northern Chile to improve copper mining processes.
\end{abstract}

\section{Introduction}

Due to the complexity of extraction mining worldwide, computer models are becoming an essential tool for reducing production costs [1]. Computational techniques have recently been incorporated into the copper industry to improve both the process and the results obtained through leaching, thus achieving tangible results such as higher levels of production, identification of low grade ore, and reduction of production costs [2].

Copper mining in Chile is the most profitable industry and contributes to approximately $12 \%$ of world copper production $[3,4]$. In recent years, the mining industry in Chile has begun to utilize and show an increasing interest in Artificial Intelligence techniques in processes such as copper production predictions, where the traditional methods tend to lack certainty and knowledge, and one of these techniques is softcomputing [5].

Softcomputing is the branch of Artificial Intelligence that groups paradigms and techniques working with incomplete and imprecise information in critical processes, in order for the company to obtain useful solutions for tasks such as prediction or discovery of information or knowledge [4-6]. A definition of softcomputing is "an emerging approach to computing which parallels that remarkable ability of the human mind to reason and learn in an environment of uncertainty and imprecision" [7].

This paper describes the work done with ANN to generate behavior predictions of the pile in the copper leaching domain. The use of ANNs has been used in combination with other techniques commonly used as a predictor in the mining industry, such as linear regression, with the aim of contrasting and enhancing the quality of results. An ANN can be defined as a set of computational units (neurons) that are highly interconnected. Each neuron is also called node which represents the biological neuron, and the connection among them represents the biological neuronal network [8]. The basic element of data processing in a neural network is the perceptron, a simple element that with an input vector provides a single output, for which it uses an activation 
function. An extended and well-accepted way to classify ANN is according to architecture and learning. Architecture refers to the arrangement and connections between neurons, number of input and output variables, and number of hidden layers, while learning classification refers to training the network by way of patterns, and the patterns are performed iteratively until the restriction $\left|O_{d}-O_{i}\right| \leq \delta$ is satisfied, $O_{d}$ being the desired output, $O_{i}$ the output of the $n$th iteration, and $\delta$ a small numerical value $[9,10]$.

This paper describes the copper recovery prediction process for an extraction mine in northern Chile, using both a statistical model (methods traditionally used in the copper leaching industry) and a neural model known as multilayer perceptron with backpropagation [11, 12]. The multilayer perceptron model is considered the most widely used neural network due to its efficiency and ease of understanding and interpretation of both the network and its results. The parameters of entry to the ANN were selected with respect to the objectives and conditions of the company. Specifically, this paper reflects the processes and results obtained in the SCM Franke Company, of the international group KGHM International, which has been operating in Chile since 2009.

\section{Background and Related Works}

The process of applying different techniques to adjust the weights related to each of the input variables of neurons in an ANN is known as learning $[8,13]$. For this process, which is also known as network training, several techniques can be followed but the most common ones are as follows: supervised and unsupervised training [14]. The supervised training consists of using a series of pairs $\left(I_{i}, O_{i}\right)$ where $I_{i}$ represents an input data vector and $O_{i}$ represents the desired output vector. The training consists of using an algorithm to make the values of $I_{i}$ as close as possible to the vector $O_{i}$, while in the unsupervised training only the vector $I_{i}$ is available and the training algorithm attempts to find the hidden structures between these data to adjust the weights of each neuron in the network [15]. In both cases, the learning rules act by modifying the weights to achieve the goal, for which there are several techniques as well. One of the most used techniques is error correction via adjustment of weights that essentially consists of obtaining a value $\delta$ very close to 0 .

Currently, the applications that include ANN are in all areas of science and engineering, that is, energy production estimation systems [16]. In engineering in particular, the increase in ANN usage in recent decades is significant, being applied to tasks such as prediction and regression. For example, in [17] a comparison between ANN and regression analysis is described, in terms of notation and implementation of both paradigms, and also highlights the advantages in terms of implementation. In $[4,18]$ works are presented related to metal-mechanical industry that compares the performance of the Bayesian networks with ANNs in the prediction of surface quality in high-speed machining processes. The most frequently used techniques for this process are ANN [13] and linear or multiple regression methods [14]. In [19], ANNs are used to predict the influence of biological and nonbiological parameters on the precipitation of ferric iron through a bioleaching process. In [20] a process of construction of a three-layer backpropagation ANN to predict the concentration of heavy metals $(\mathrm{Zn}, \mathrm{Ni}, \mathrm{Cd}$, and $\mathrm{Pb}$ ) as waste in a zinc leach cell is described.

In [21] an ANN used to predict the copper production process is presented as well. Moreover, in the work recently reported in [22] an ANN is used to predict copper flotation rates under different operating conditions; different dosages of chemical reagents used in the process, feed rate, and granulometry are used for this process. A three-layer backpropagation neural network (input layer, two hidden layers, and one output), topology (9-10-10-3), was used, and the quality of the prediction in the testing process was $93 \%$.

\section{Materials and Methods}

The SCM Franke Company uses three industrial processes widely known in the copper industry to produce metallic copper via hydrometallurgy: leaching in dynamic cells, solvent extraction, and electrowinning. The goal in these processes is to achieve the highest copper production by saving resources and having the lowest possible environmental impact. In this sense, the company considered carrying out simulations to predict the copper production through leaching processes. The objective was to produce an efficient model (with an accuracy greater than 95\%) for the estimation of copper leaching recovery based on historical data.

A previous analysis by the SCM Franke Company conducted to determine which of these processes is the least controlled (number of influencing factors and homogeneity of irrigation) found it to be the leaching process in dynamic stacks. Based on this previous analysis, the parameters to be used in the prediction models and the desired quality level (95\%) were identified and employed as the desired adjustment value in the simulation to test the results of the models.

The study summarized the prediction of copper extraction in dynamic stacks, applying mathematical models (statistical model) that require complete information to give precise results and an ANN, which is considered to have advantages over the treatment of incomplete information in terms of generating predictions in industrial production.

Pile leaching copper is a percolation process that operates above ground. The procedure is illustrated in Figure 1. The oxide copper ore is piled up on leach pads which have an inclination of $3^{\circ}$ approx. and a rubber lining that seals the ground beneath. These heaps are 3-meter-high and 72 meters in length with a base area of 2,880 square meters, covering 120,000 tons of ore.

A sprinkler system is installed on top of each heap allowing diluted sulfuric acid to be fed uniformly over the ore. While the solution percolates through the pile, copper is leached out of the ore. The pregnant leaching solution is collected by a drainage system below the pile and is led through a collection ditch into a pond.

\section{Methodology}

The research consists of two stages, the first called "copper recovery modeling" in which models are generated with each 


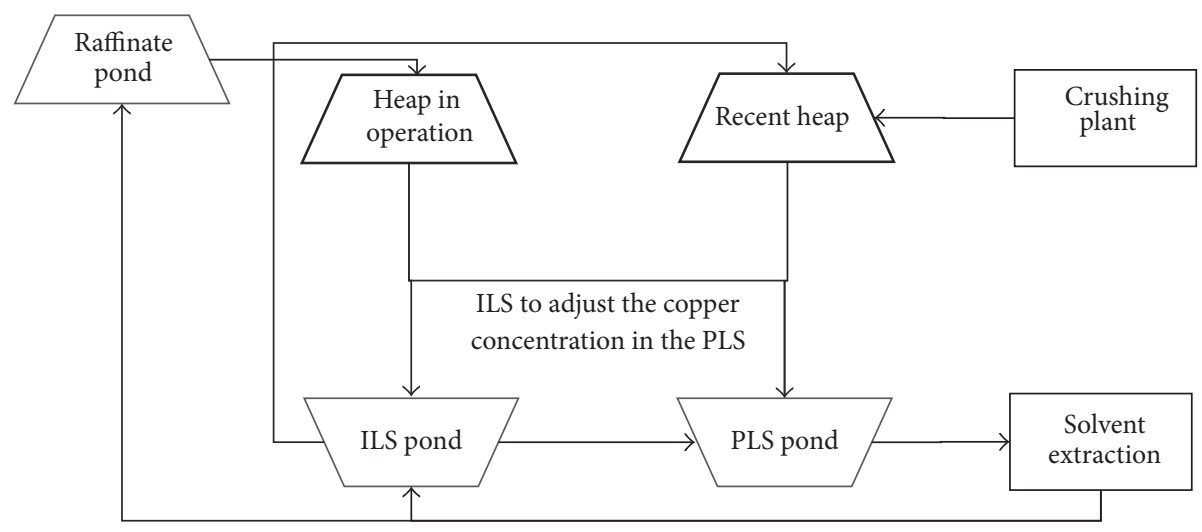

FIGURE 1: Process flow diagram of the pile leaching process.

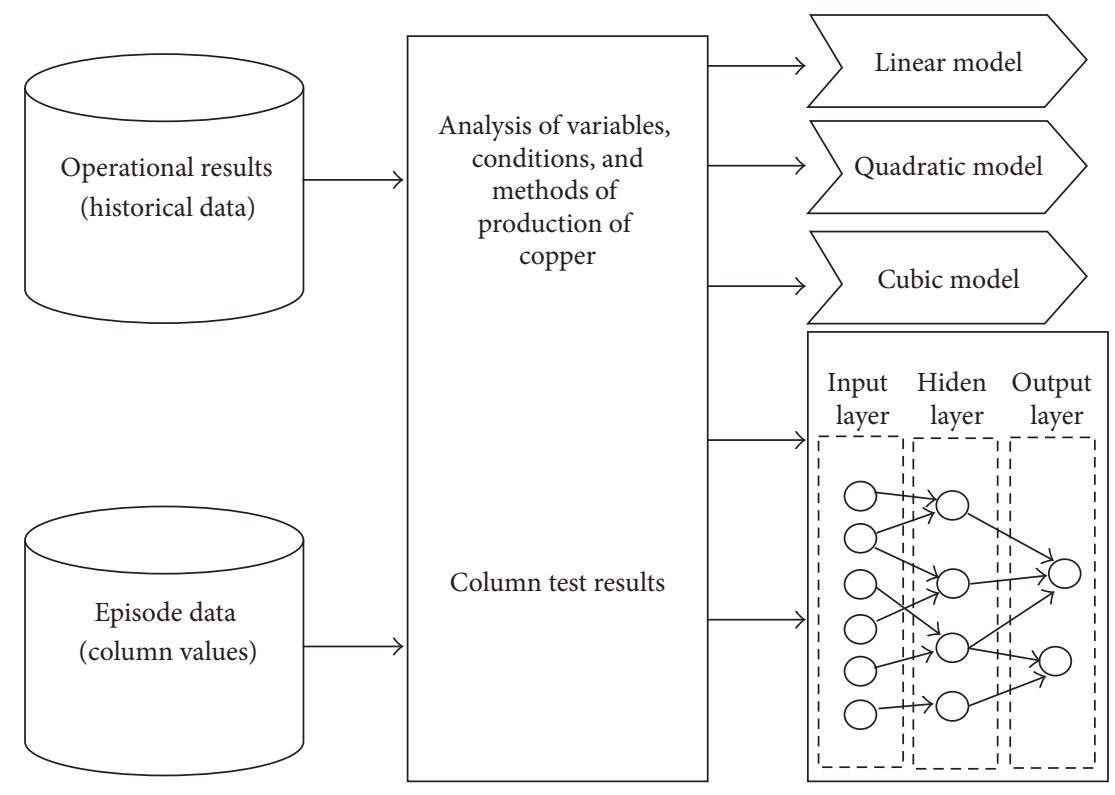

FIGURE 2: Work scheme for copper recovery prediction in dynamic stacks.

of the aforementioned methods and the second stage called "Evaluation" where the results are compared and the quantity and quality of information are obtained.

Both stages are beneficial for the SCM Frank Company in order to understand the leaching process with the characteristics of the ore and to be able to predict the recovery of copper in dynamic stacks, focused on achieving a less than $5 \%$ margin of error in estimation. These two stages are possible due to the previous analysis and selection of variables and parameters representing the inputs to the prediction methods; Figure 2 graphically shows this work scheme.

4.1. Statistical Models. In order to perform the modeling, the most recent literature in leaching process was considered, taking into account the variables that affect the recovery of copper. In addition, the historical operational results data and process-related pilot testing were taken into account, which we call pilot data. Pilot tests consist of testing columns with strict control measures regarding irrigation rates, acid concentrations in irrigation solutions, and operating cycles, conditions that vary depending on the test to be performed. A case study was generated with a database of approximately 30,000 pieces of data.

4.1.1. Data Collection. For the purpose of this study, the historical database corresponding with industrial and piloting performance from SCM Franke Company was used. The data stored in the database correspond to both plant data operation (weighted values and accumulated daily) and pilot data.

The operating plant data were obtained at a frequency of 4 hours for 1 year. For some periods, the irrigation was stopped on some cells or modules in service; due to these periods, inconsistent results were disregarded and corresponding data was not considered for the data collection process; some "noise" in the system and useless information were 
TABLE 1: Entry parameters to the statistical model.

\begin{tabular}{lcc}
\hline Notation & Name and description & Optimum values \\
\hline$x_{1}$ & $\begin{array}{c}\text { Monoclass granulometry (refers } \\
\text { to the mineral) }\end{array}$ & Between $11,5 \mathrm{~mm}$ and $15 \mathrm{~mm}$ \\
$x_{2}$ & Irrigation rates & Between $14(1 \times \mathrm{h}) / \mathrm{m}^{2}$ and 6 \\
$(1 \times \mathrm{h}) / \mathrm{m}^{2}$ & Between $0,5 \mathrm{~g} / \mathrm{l}$ and $100 \mathrm{~g} / \mathrm{l}$ \\
$x_{3}$ & Total acid added & Between $1 \mathrm{~m}$ and $5 \mathrm{~m}$ \\
$x_{4}$ & Pile high & Between $0,5 \%$ and $2 \%$ \\
$x_{5}$ & Total copper grade & Between $0,5 \%$ and $10 \%$ \\
$x_{6}$ & $\mathrm{CO}_{3}$ grade & Less than $15 \mathrm{~m}^{3} / \mathrm{TMS}$ in pilot and \\
$x_{7}$ & Leaching ratio & Less than $8 \mathrm{~m}^{3} / \mathrm{TMS}$ in plant \\
$x_{8}$ & Operation days & Between 90 and 120 days \\
$x_{9}$ & Soluble copper grade stacked & $70 \%$ of the total copper grade \\
\hline
\end{tabular}

TABLE 2: Comparative of $R^{2}$ values.

\begin{tabular}{lc}
\hline Model & $R^{2}$ value \\
\hline Lineal model & $69,8 \%$ \\
Quadratic model (standard deviation $=9,4)$ & $89,9 \%$ \\
Cubic model (standard deviation $=8,3)$ & $92,3 \%$ \\
ANN model & $97,9 \%$ \\
\hline
\end{tabular}

disregarded as well. Regarding the pilot data, the method of information collection was the same used for the data of operation.

The next step was to identify the parameters that affect the copper recovery and to form a robust database with this information for use in the preparation and evaluation of process models using different techniques in order to determine one that meets the plant requirements (adjustment greater than 95\%) with respect to plant and pilot operating data. The parameters considered in the statistical model are detailed in Table 1.

A search was performed to find the combination of suitable variables to produce the most successful model for the desired response. The system response was defined as the percentage of copper extraction. To validate the model, the response variable was monitored. The model was monitored in order to obtain the best fit for the operating conditions and variations. When model misalignment was below acceptable (much lower than the desired 95\%), the model was readjusted and remade considering the operating ranges that were initially not considered; parameters that were initially not considered were now carried out.

To select the subset of variables that ensure a practical model, coefficients were generated using the Minitab software. In detail, the calculated coefficients were as follows: Vars, $R$-cuad, adjusted $R$-cuad, $C$ p of Mallows, and $S$ (standard dispersion). A preliminary statistical model was made considering the Press factor, used to avoid overadjustment. Additionally, for this model the following restrictions were considered: (1) that the adjusted values of $S, R$-cuad $\left(R^{2}\right)$, and $R$-squared were the highest possible and (2) that the value of the Press factor was the highest grade possible.
Originating from the preliminary model and utilizing the Minitab tools, three statistical models were generated: linear adjustment model, quadratic adjustment model, and cubic adjustment model. Table 2 summarizes each of these models and the adjustments results according to the value $R^{2}$.

For the lineal model (1),

$$
Y=\sum_{i=1}^{9} \alpha_{i} * \chi_{i}+C
$$

For the quadratic and cubic models (2)

$$
\begin{aligned}
Y= & \sum_{i=1}^{9} \alpha_{i} * \chi_{i}+\sum_{j=1}^{9} \sum_{i=1}^{9} b_{i} * \chi_{i} * \chi_{j}+C, \\
Y= & \sum_{i=1}^{9} a_{i} * \chi_{i}+\sum_{j=1}^{9} \sum_{i=1}^{9} b_{i} * \chi_{i} * \chi_{j} \\
& +\sum_{k=1}^{9} \sum_{j=1}^{9} \sum_{i=1}^{9} c_{i} * \chi_{i} * \chi_{j} * \chi_{k}+C
\end{aligned}
$$

where

(i) $\chi_{i}, \chi_{j}$, and $\chi_{k}$ represent the input value of the variable;

(ii) $a_{i}, b_{i}$, and $c_{i}$ represent the weight of the input;

(iii) $C$ represents a constant

4.2. ANN Model Base. To perform the RNA modeling, the MATLAB program was applied. For the network programming, the following parameters were used: 9 income variables 
(see Table 1), hidden layers, $60 \%$ of the information for the network, $40 \%$ of the information for network validation, 0.2 of error as cycle-error, or maximum of 500 iterations. The data for the training and validation was randomly selected.

In the variables selection the following was considered: the experience accumulated by operation experts of the SCM Franke Company, the results of the piloting, bibliography, and the results observed with the statistical models described above. In the ANN configuration, nine input variables were used (see Table 1) for a multilayer perceptron model with backpropagation algorithm.

The output layer was formed by a corresponding neuron to the prediction of copper recovery in dynamic piles, equally focusing on achieving a less than $5 \%$ error in the prediction estimation. The mathematical expression used in each neuron to obtain the output value is indicated in (3) where $\left(x_{i}\right)$ represents the input value to the neuron and the variable $\left(w_{i j}\right)$ represents the weight associated with the neuron. The task of initializing the weights associated with each input variable was performed randomly in a range normalized between $(-1,1)$ and using the MAPMINMAX function of MATLAB (a function used to normalize the input and output values of the neurons), and threshold values that were used in the activation functions (sigmoid function) were initialized in a similar way (these data were not reflected in this document because they are subject to confidentiality agreements with the company). The mathematical expression of the sigmoid function is indicated in

$$
y=\sum_{j=1}^{9} L_{j} \sum_{i=9}^{9} w_{i j}^{i} * x_{i},
$$

where

(i) $x_{i}$ represents the $i$ th input value to the neuron;

(ii) $w_{i j}^{i}$ represents the $i$ th weight associated with the neuron $i$ of the layer $j$;

(iii) $L_{j}$ represents the constant of the $j$ th layer.

\section{Results}

Table 2 shows the $R^{2}$ values obtained by both, algorithmic models and ANN model; as seen in the table, the second-best value of $R^{2}$ corresponds to the cubic fit model. None of these values exceed the desired minimum value (95\%); however, the values obtained in the simulation, using cubic algorithmic models, are close to what was obtained.

The best value corresponding to the ANN model during the learning phase and validation of the ANN, a $97,9 \%$ of adjustment, was obtained. Figure 3 shows the adjustment performed with the ANN.

\section{Discussion}

The result obtained through linear modeling was far from the required $(89.9 \% \mathrm{v} / \mathrm{s} 95 \%)$, which indicates that the capacity to make the needed adjustment far exceeds what can be delivered by this type of modeling. The adjustment results

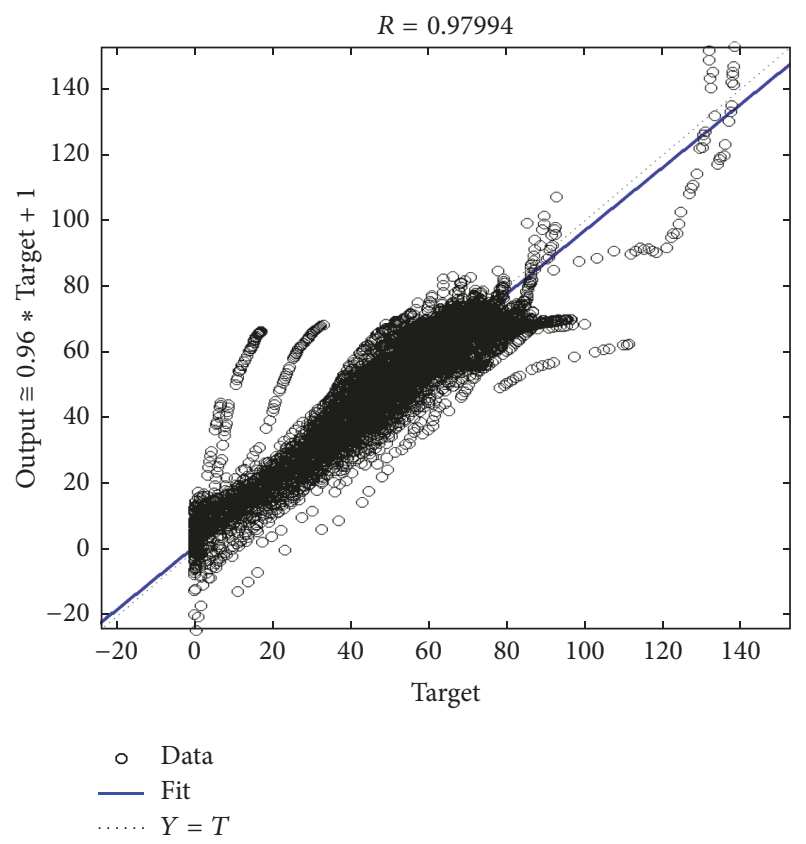

FIGURE 3: ANN validation model.

of the quadratic model were approximately $22 \%$ better than those obtained previously and the $R^{2}$ value was very similar to $R^{2}$ (adjusted), which means the quadratic model is better able to generate more realistic data projections. With respect to the cubic model, the observed adjustment was close to what was obtained using the quadratic model, which indicates that the use of higher-grade models does not present a significant improvement in relation to the model adjustment, due to the limitations given by the techniques used and the high variability presented by the data used.

The use of ANN for system modeling led to an improvement from $92.3 \%$ to $97.9 \%$, compared to the cubic model. It was observed that the use of ANN achieved the objective of adjusting to fit, which was due to the complex network that this process uses to adjust the system response to the existing parameters.

To achieve these results through the use of ANN, the use of a program such as MATLAB or another advanced calculation program is required. Due to the high complexity of such a modeling technique, it requires a database with an abundance of information so as to give the program the information necessary to evaluate as many cases as possible. On the other hand, ANN has the disadvantage of not being easily detected compared to statistical models, so this could present a problem for new users.

\section{Conclusion}

SCM Franke has carried out simulations on other copper production processes and knows from experience that the prediction is much cheaper than the experimental work, but, in this case, our study describes a prediction model that represents the behavior of the leaching plant, considering the 
variables initially defined with adjustment results higher than 95\% obtained.

Accordingly, it was determined that ANN was the best model for SCM Franke mining leach plant, due to the high variability of the existing plant results and tests. The adjustment obtained was $97.9 \%$, which is higher than the adjustment of $95 \%$ initially requested.

This study has served to obtain a comparison between prediction models and it can be intuited by the precision of the adjustments that the neural model has potential for use in future copper production prediction process. Furthermore, experience was gained in defining the model of an ANN, which can be used in future process simulations related to the improvement of copper attainment via softcomputing techniques in the SCM Franke Company or in similar companies.

\section{Conflicts of Interest}

The authors declare that they have no conflicts of interest.

\section{Acknowledgments}

Thanks are due to SCM Franke for supporting this project, specifically for the collaboration on data provision and storing and for the experience made available to the authors to select the parameters and criteria used for modeling.

\section{References}

[1] M. Milivojevic, S. Stopic, B. Friedrich, B. Stojanovic, and D. Drndarevic, "Computer modeling of high-pressure leaching process of nickel laterite by design of experiments and neural networks," International Journal of Minerals, Metallurgy and Materials, vol. 19, no. 7, pp. 584-594, 2012.

[2] H. Kamran Haghighi, D. Moradkhani, and M. M. Salarirad, "Modeling of synergetic effect of LIX $984 \mathrm{~N}$ and D2EHPA on separation of iron and zinc using artificial neural network," Transactions of the Indian Institute of Metals, vol. 67, no. 3, pp. 331-341, 2014.

[3] ICSG PRESS RELEASE. Copper: Preliminary Data for September 2013, 2013.

[4] V. Flores, Y. Hadfeg, J. Bekios, A. Quelopana, and C. Meneses, "A method for automatic generation of explanations from a rule-based expert system and ontology," Advances in Intelligent Systems and Computing, vol. 537, pp. 167-176, 2017.

[5] L. A. Zadeh, "Fuzzy logic, Neural networks, and soft computing," Communications of the ACM, vol. 37, no. 3, pp. 77-84, 1994.

[6] L. G. Bergh, J. B. Yianatos, and C. A. Leiva, "Fuzzy supervisory control of flotation columns," Minerals Engineering, vol. 11, no. 8, pp. 739-748, 1998.

[7] L. A. Zadeh, "Soft computing and fuzzy logic," IEEE Software, vol. 11, no. 6, pp. 48-56, 1994.

[8] J. J. Hopfield, Artificial Neural Networks, vol. 4, Berlin, 1988.

[9] A. J. Maren, C. T. Harston, and R. M. Pap, Handbook of Neural Computing Applications, San Diego, Calif, USA, 2014.

[10] A. Freeman James and M. Skapura David, Neural Networks: Algorithms, Applications and Programming Techniques, Addison-Wesley, New York, NY, USA, 1991.

[11] M. Cilimkovic, "Neural Networks and Back Propagation Algorithm," FettTu-SofiaBg, 2010.
[12] V. Skorpil and J. Stastny, "Neural networks and back propagation algorithm," Electron Bulg Sozopol, pp. 20-22, 2006.

[13] S. S. Lee and J. C. Chen, "On-line surface roughness recognition system using artificial neural networks system in turning operations," The International Journal of Advanced Manufacturing Technology, vol. 22, no. 7-8, pp. 498-509, 2003.

[14] C.-X. Feng and X.-F. Wang, "Surface roughness predictive modeling: Neural networks versus regression," Institute of Industrial Engineers (IIE). IIE Transactions, vol. 35, no. 1, pp. 11-27, 2003.

[15] H.-Y. Shu, H.-C. Lu, H.-J. Fan, M.-C. Chang, and J.-C. Chen, "Prediction for energy content of taiwan municipal solid waste using multilayer perceptron neural networks," Journal of the Air \& Waste Management Association, vol. 56, no. 6, pp. 852-858, 2006.

[16] T. C. Ogwueleka and F. N. Ogwueleka, "Modelling energy content of municipal solid waste using artificial neural network," Journal of Environmental Health Science and Engineering, vol. 7, no. 3, pp. 259-266, 2010.

[17] M. Paliwal and U. A. Kumar, "Neural networks and statistical techniques: a review of applications," Expert Systems with Applications, vol. 36, no. 1, pp. 2-17, 2009.

[18] Correa M. Explanation of a Bayesian network classifier by means of decision trees. n.d.

[19] H. Golmohammadi, A. Rashidi, and S. Safdari, "Predvidstrokeanje precipitacije feri jona u procesu bioluženja primenom parcijalnih najmanjih kvadrata i veštačke neuronske mreže," Chemical Industry \& Chemical Engineering Quarterly, vol. 19, no. 3, pp. 321-331, 2013.

[20] D. E. Rumelhart, G. E. Hinton, and R. J. Williams, "Learning representations by back-propagating errors," Nature, vol. 6, pp. 323-5336, 1986.

[21] H. Kamran Haghighi, M. Rafie, D. Moradkhani, B. Sedaghat, and A. Abdollahzadeh, "Modeling on Transition of Heavy Metals from Ni-Cd Zinc Plant Residue Using Artificial Neural Network," Transactions of the Indian Institute of Metals, vol. 68, no. 5, pp. 741-756, 2015.

[22] O. Salmani Nuri, E. Allahkarami, M. Irannajad, and A. Abdollahzadeh, "Estimation of selectivity index and separation efficiency of copper flotation process using ANN model," Geosystem Engineering, vol. 20, no. 1, pp. 41-50, 2017. 

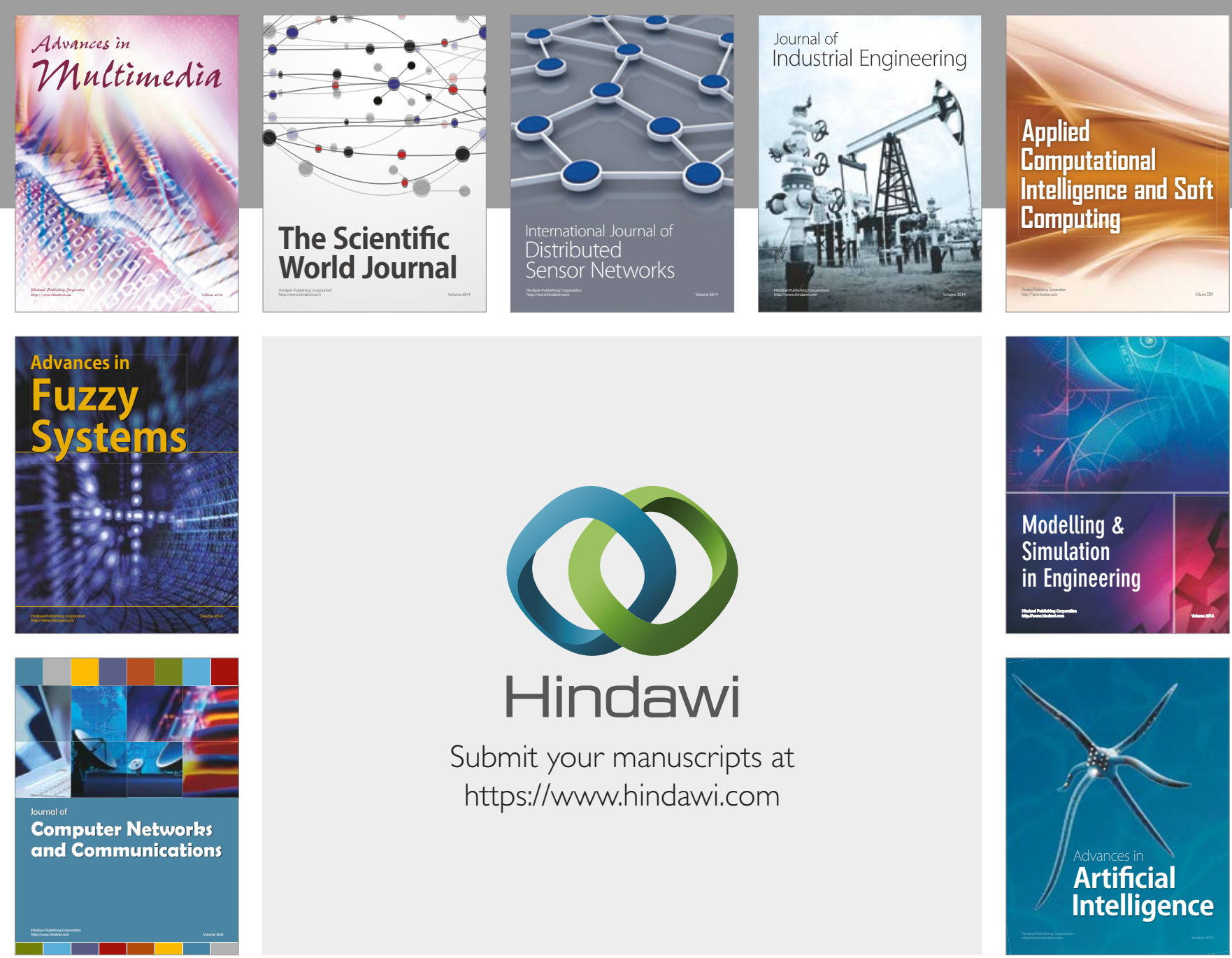

\section{Hindawi}

Submit your manuscripts at

https://www.hindawi.com
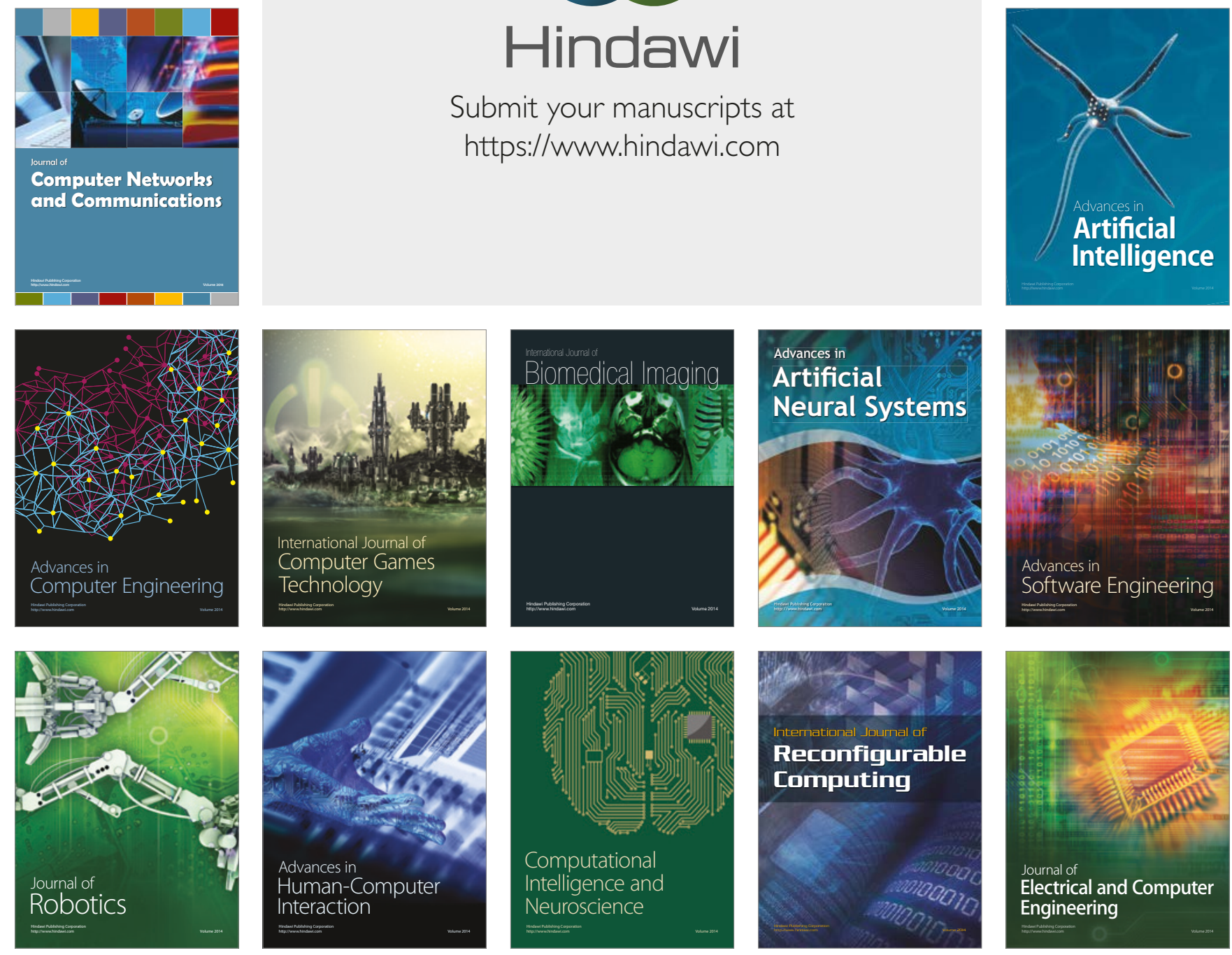